19, 129. https://doi.org/10.1186/s13059-0181509-y.

Volkova, N.V., Meier, B., González-Huici, V., Bertolini, S., Gonzalez, S., Vöhringer, H., Abascal, F., Martincorena, I., Campbell, P.J., Gartner, A., and Gerstung, M. (2020). Mutational signatures are jointly shaped by DNA damage and repair. Nat. Commun. 11, 2169. https://doi.org/10.1038/ s41467-020-15912-7.
Wojtowicz, D., Leiserson, M.D.M., Sharan, R., and Przytycka, T.M. (2020). DNA Repair Footprint Uncovers Contribution of DNA Repair Mechanism to Mutational Signatures. Pac. Symp. Biocomput. 25, 262-273.

Wojtowicz, D., Hoinka, J., Amgalan, B., Kim, Y.A., and Przytycka, T.M. (2021). RepairSig: Deconvolution of DNA damage and repair contributions to the mutational landscape of cancer. Cell Syst. 12 994-1003.

Zou, X., Owusu, M., Harris, R., Jackson, S.P., Loizou, J.I., and Nik-Zainal, S. (2018). Validating the concept of mutational signatures with isogenic cell models. Nat. Commun. 9, 1744. https://doi. org/10.1038/s41467-018-04052-8.

\title{
DeCiFering the subclonal composition of tumors
}

\author{
Haixi Yan, ${ }^{1}$ Maxime Tarabichi, ${ }^{1,2}$ Nicholas McGranahan, ${ }^{3}$ and Peter Van Loo ${ }^{1, *}$ \\ ${ }^{1}$ The Francis Crick Institute, London, UK \\ 2Institute for Interdisciplinary Research, Université Libre de Bruxelles, Brussels, Belgium \\ ${ }^{3}$ University College London Cancer Institute, London, UK \\ ${ }^{*}$ Correspondence: peter.vanloo@crick.ac.uk \\ https://doi.org/10.1016/j.cels.2021.09.008
}

Accurately identifying the subclones that make up tumors is critical for understanding cancer biology. In an article in this issue of Cell Systems, Satas et al. examine mutations with an evolutionary perspective to decipher the composition of tumors.

Cancers acquire mutations during their development and progression. Single nucleotide variants (SNVs) are common and are thought to play a key role in driving tumorigenesis by enabling cancer hallmarks and providing selective advantages. In addition to being used to study the functional impact of these mutations, SNVs can be used as genetic fingerprints to trace the lineage of different tumor clones. As most SNVs are retained by descendants of their common ancestor cell, we can cluster SNVs by the inferred fraction of cells carrying them, the so-called cancer cell fraction (CCF). The CCF is a central concept that allows us to identify clones and place them in relation to each other and thereby reconstruct the most likely phylogenetic tree of a tumor (Tarabichi et al., 2021). These phylogenetic trees have given us vital insights into how tumors develop and are expected to inform clinical decision making (Turajlic et al., 2018).

Inferring the CCF of an SNV requires estimation of the number of DNA copies carrying it, or its multiplicity. To date, most scalable methods have relied on the assumption that an SNV has the same multiplicity in all tumor cells in a sample (Roth et al., 2014; Tarabichi et al., 2021), referred to as the constant mutation multiplicity. However, this assumption is often violated. Indeed, cancers are inherently heterogeneous with most tumors containing genetically distinct subclones (Dentro et al., 2021). These subclones can have distinct copy number profiles, resulting in different multiplicity values for the same SNV.

A key limitation of reconstructing a cancer's evolutionary history is that we usually have a single time point, i.e., we do not have a fossil record-we only have the extant species to investigate. Thus, we cannot acquire information from chromosomes lost by tumor cells, such as during loss of heterozygosity events. Previous methods either did not account for this limitation or only partially did so, typically through inelegant hacks. To address this, Satas et al. (2021) propose the interesting concept of a descendant cell fraction (DCF). While the CCF is the proportion of cells carrying the SNV, the DCF is the proportion of cells that either themselves have the SNV or whose ancestors carried the SNV. For mutations on chromosomes that were present in an ancestral clone but were subsequently lost during evolution, CCF-based methods would errone- ously infer the mutations to be subclonal (Figure 1A). In contrast, the DCF statistic formally accounts for this subsequent loss (Figure 1B). Thus, DCF allows the recovery of this fossil information.

Satas et al. (2021) have implemented these concepts in a novel algorithm called DeCiFer to improve estimation of CCFs in tumor samples. DeCiFer assesses potential genotypes of subclones detected through copy number changes by subjecting different pairs of multiplicities to evolutionary constraints. It then jointly optimizes the mutation multiplicities across subclones and the mutations' DCFs, resulting in more accurate subclonal reconstruction. Computational approaches that accurately detect subclonal copy number changes are a critical input for methods such as DeCiFer (Carter et al., 2012; NikZainal et al., 2012; Zaccaria and Raphael, 2020). This is an active area of research and as these methods for detecting subclonal copy number changes improve, so too will the accuracy of DCFs and clusters generated by DeCiFer.

In order to assess the benefits of the DeCiFer approach in a real-world multisample dataset, Satas et al. generated DCFs for all SNVs in a published dataset of 10 patients with metastatic prostate 


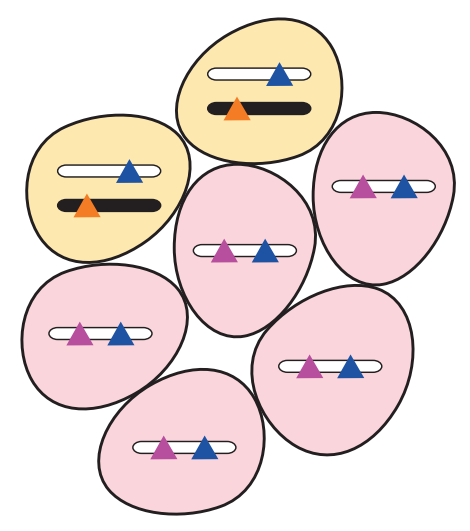

A

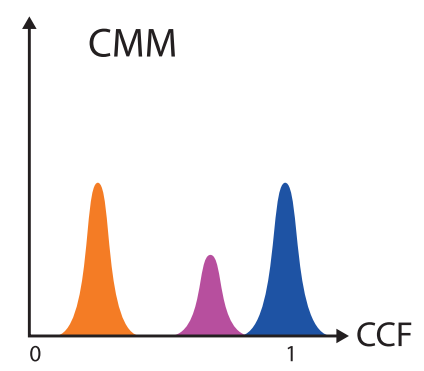

B

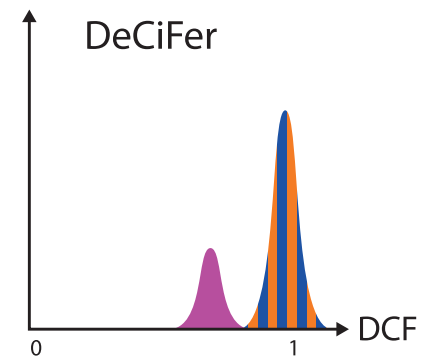

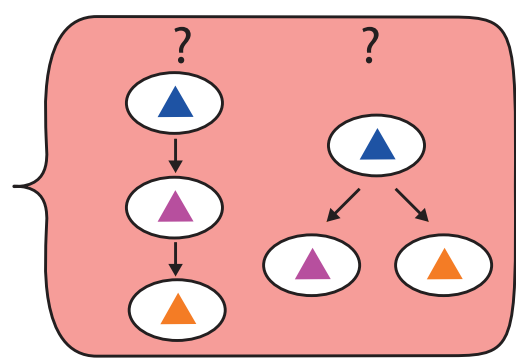

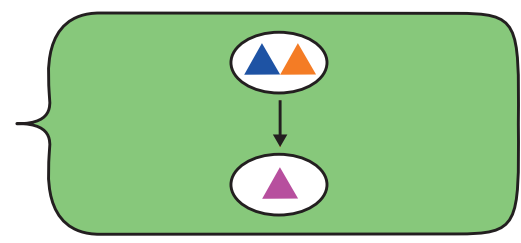

Figure 1. Reconstructing tumor evolution using mutations

(A) A constant mutation multiplicity (CMM) assumption may lead to incorrect interpretation of CCF values due to subclonally lost mutations.

(B) In contrast, by considering DCFs, DeCiFer generates more plausible phylogenetic trees.

cancer (Gundem et al., 2015). They compared the DCFs generated by DeCiFer to CCFs generated using a constant mutation multiplicity assumption. This allowed them to highlight several interesting scenarios where DeCiFer was able to reassign SNVs, leading to biologically more likely interpretations of the data. Indeed, reconstruction methods generally do not consider which allele mutations lie on and whether the steps implied by the reconstructed trees are biologically plausible. By applying different multiplicities to two subclones, DeCiFer was able to calculate DCFs of truncal SNVs that were otherwise classified as subclonal due to an incorrect mutation multiplicity being applied to one subclone. Furthermore, by using the DCF, SNVs lost in a subclonal copy-neutral loss of heterozygosity event were correctly classified as truncal. Although there was no ground truth for the phylogeny of these tumors, the more parsimonious solutions generated by DeCiFer appear more likely and help resolve some of these evolutionary histories.

Cancer evolution is a dynamic process, and this study highlights the important interplay between SNVs and copy number changes. Although perhaps counterintuitive, mutations that are present in the most recent common ancestor may not remain clonal later in evolution, as a descendant subclone may lose the chromosomes carrying those mutations. In addition to its importance for reconstructing tumor evolution, this phenomenon also has key treatment implications. Targeting clonal actionable mutations or clonal neoantigens, strategies that have received much recent research interest, would need to account for this. One could hypothesize that optimal treatment strategies would likely need to target mutations that, due to evolutionary and biological constraints, are unlikely to be lost by copy number changes (McGranahan and Swanton, 2019).

Ultimately, high-throughput, robust, and error-free single-cell DNA sequencing approaches will allow much more detailed resolution of tumor heterogeneity. However, such approaches are still in their infancy, and large cohorts and multi-sample studies of tumors are likely to be profiled using bulk sampling in the years to come. DeCiFer joins a suite of tools that enable more accurate deconvolution of individual tumors, allowing us to derive new insights into tumor evolution.

\section{ACKNOWLEDGMENTS}

This work was supported by the Francis Crick Institute, which receives its core funding from Cancer
Research UK (FC001202), the UK Medical Research Council (FC001202), and the Wellcome Trust (FC001202). M.T. was supported as a postdoctoral researcher of the F.R.S.-FNRS. N.M. is a Sir Henry Dale Fellow, jointly funded by the Wellcome Trust and the Royal Society (Grant Number 211179/Z/18/Z), and receives funding from Cancer Research UK Lung Cancer Centre of Excellence, Rosetrees and the NIHR BRC at University College London Hospitals.

\section{DECLARATION OF INTERESTS}

N.M. has received consultancy fees from Achilles Therapeutics.

\section{REFERENCES}

Carter, S.L., Cibulskis, K., Helman, E., McKenna, A., Shen, H., Zack, T., Laird, P.W., Onofrio, R.C. Winckler, W., Weir, B.A., et al. (2012). Absolute quantification of somatic DNA alterations in human cancer. Nat. Biotechnol. 30, 413-421.

Dentro, S.C., Leshchiner, I., Haase, K., Tarabichi, M., Wintersinger, J., Deshwar, A.G., Yu, K., Rubanova, Y., Macintyre, G., Demeulemeester, J., et al.; PCAWG Evolution and Heterogeneity Working Group and the PCAWG Consortium (2021). Characterizing genetic intra-tumor heterogeneity across 2,658 human cancer genomes. Cell 184, 2239-2254.e39.

Gundem, G., Van Loo, P., Kremeyer, B., Alexandrov, L.B., Tubio, J.M.C., Papaemmanuil, E., Brewer, D.S., Kallio, H.M.L., Högnäs, G. Annala, M., et al.; ICGC Prostate Group (2015). The evolutionary history of lethal metastatic prostate cancer. Nature 520, 353-357.

McGranahan, N., and Swanton, C. (2019) Neoantigen quality, not quantity. Sci. Transl. Med. 11, eaax7918. 


\section{Previews}

Nik-Zainal, S., Van Loo, P., Wedge, D.C., Alexandrov, L.B., Greenman, C.D., Lau, K.W., Raine, K., Jones, D., Marshall, J., Ramakrishna, M., et al.; Breast Cancer Working Group of the International Cancer Genome Consortium (2012). The life history of 21 breast cancers. Cell 149 994-1007.

Roth, A., Khattra, J., Yap, D., Wan, A., Laks, E., Biele, J., Ha, G., Aparicio, S., Bouchard-Côté, A., and Shah, S.P. (2014). PyClone: statistical inference of clonal population structure in cancer. Nat. Methods 11, 396-398.
Satas, G., Zaccaria, S., El-Kebir, M., and Raphael, B.J. (2021). DeCiFering the elusive cancer cell fraction in tumor heterogeneity and evolution. Cell Syst. 12, 1004-1018.

Tarabichi, M., Salcedo, A., Deshwar, A.G., N Leathlobhair, M., Wintersinger, J., Wedge, D.C. Van Loo, P., Morris, Q.D., and Boutros, P.C. (2021). A practical guide to cancer subclonal reconstruction from DNA sequencing. Nat. Methods 18, 144-155.
Turajlic, S., Xu, H., Litchfield, K., Rowan, A. Horswell, S., Chambers, T., O'Brien, T., Lopez, J.I., Watkins, T.B.K., Nicol, D., et al.; TRACERx Renal Consortium (2018). Deterministic Evolutionary Trajectories Influence Primary Tumor Growth: TRACERx Renal. Cell 173, 595610.e11.

Zaccaria, S., and Raphael, B.J. (2020). Accurate quantification of copy-number aberrations and whole-genome duplications in multi-sample tumor sequencing data. Nat. Commun. $11,4301$. 